\title{
Mesenchymal stem cell-derived extracellular vesicles prevent neural stem cell hypoxia injury via promoting miR-210-3p expression
}

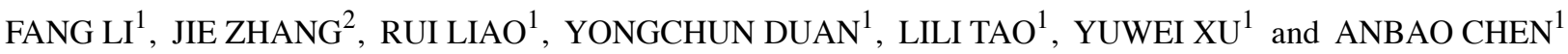 \\ ${ }^{1}$ Department of Emergency Internal Medicine, The Second Affiliated Hospital of Kunming \\ Medical University, Kunming, Yunnan 650101; ${ }^{2}$ Organ Transplantation Center, The First Affiliated \\ Hospital of Kunming Medical University, Kunming, Yunnan 650032, P.R. China
}

Received October 24, 2019; Accepted May 29, 2020

DOI: $10.3892 / \mathrm{mmr} .2020 .11454$

\begin{abstract}
Neural stem cells (NSCs) have the potential to give rise to offspring cells and hypoxic injury can impair the function of NSCs. The present study investigated the effects of mesenchymal stem cell (MSC)-derived extracellular vesicles (EVs) on NSC injury, as well as the underlying mechanisms. MSC-EVs were isolated and identified via morphological and particle size analysis. Cobalt chloride was used to establish a hypoxic injury model in NSCs. Terminal deoxynucleotidyl transferase dUTP nick end labeling assay was conducted to detect apoptosis. Reverse transcription-quantitative PCR was performed to detect the expression levels of miR-210-3p, and western blotting was used to detect the expression levels of apoptosis-inducing factor (AIF) and Bcl-2 $19 \mathrm{kDa}$ interacting protein (BNIP3). Compared with the control group, NSC apoptosis, and the expression of miR-210-3p, AIF and BNIP3 were significantly higher in the cobalt chloride-induced hypoxia group. By contrast, treatment with MSC-EVs further increased miR-210-3p expression levels, but reduced NSC apoptosis and the expression levels of AIF and BNIP3 compared with the model group $(\mathrm{P}<0.05)$. In addition, miR-210-3p inhibitor reduced miR-210-3p expression, but promoted hypoxia-induced apoptosis and the expression levels of AIF and BNIP3 compared with the model group $(\mathrm{P}<0.05)$. Collectively, the results suggested that MSC-EVs prevented NSC hypoxia injury by promoting miR-210-3p expression,
\end{abstract}

Correspondence to: Dr Jie Zhang, Organ Transplantation Center, The First Affiliated Hospital of Kunming Medical University, 295 Xichang Road, Kunming, Yunnan 650032, P.R. China E-mail: jiezhang119@126.com

Dr Anbao Chen, Department of Emergency Internal Medicine, The Second Affiliated Hospital of Kunming Medical University, 374 Dianmian Avenue, Kunming, Yunnan 650101, P.R. China

E-mail: anbaochen11@163.com

Key words: extracellular vesicles, microRNA-210-3p, mesenchymal stem cells, neural stem cells which might reduce AIF and BNIP3 expression levels and NSC apoptosis.

\section{Introduction}

Neural stem cells (NSCs) are able to self-renew and differentiate into neurons, oligodendrocytes and astrocytes under appropriate inducing conditions (1); therefore, NSCs are the most commonly used stem cells in repairing neuronal injuries (2). In neurogenesis, NSCs exist in the developing brain and serve an important role in brain plasticity throughout life $(3,4)$. Transplanting NSCs into the host brain is an efficient strategy to repair neuronal injury and treat neurodegenerative diseases (5).

Mesenchymal stem cells (MSCs) have the potential of multidirectional differentiation and neuroprotective effects (6). Notably, it may be the secretory products rather than the stem cells themselves, which serve a dominant role in neuroprotection (7-10). Extracellular vesicles (EVs) of MSCs have advantages, including being more efficient when used in the treatment of diseases compared with MSCs (11-13). EVs carry a variety of proteins, lipids, RNAs, microRNAs (miRs) and other molecules, and have a high ability to enter into target cells $(14,15)$. Among the cargo of EVs, miRs are critical posttranscriptional factors that regulate biological and physiological functions (16). A large number of different miRs are expressed sequentially and participate in the process of nerve repair or promote neuron regeneration following peripheral nerve injury (17). miR-210-3p is a key regulator that determines neuron survival under hypoxic conditions (18). miR-210-3p inhibits apoptosis and promotes cell survival under oxygen-glucose deprivation and oxidative stress conditions, and repairs neurons following spinal cord injury (19). In addition, miR-210-3p is present in EVs, which indicates a possible physiological function for miR-210-3p (20).

Cobalt chloride is widely used to produce in vitro hypoxic injury models in NSCs $(21,22)$ as it triggers NSC apoptosis via the nuclear factor erythroid 2-related factor 2 (Nrf2)/antioxidant response elements (ARE) signaling pathway (21). The present study aimed to examine the potential function of 
miR-210-3p in the protective effects of MSC-EVs against NSC hypoxic injury regarding NSC apoptosis.

\section{Materials and methods}

Animals. A total of 5 pregnant Sprague-Dawley (SD) rats (350 g, aged 3 months) were purchased from Hunan Slake Jingda Laboratory Animal Co., Ltd. (license no. SCXK (Hunan) 2016-0002). The animals were housed in a specific pathogen-free environment at $23 \pm 2^{\circ} \mathrm{C}$ with $45-65 \%$ humidity, 12-h light/dark cycles and free access to food and water. All experimental protocols were approved by the Ethics Committee of Kunming Medical University.

Preparation of EVs from MSCs. MSCs were purchased from BeNa Culture Collection (cat no. BNCC 340370; Suzhou Bena Chuanglian Biotechnology Co. Ltd.). After 48-h starvation using fetal bovine serum (FBS)-free Cellartis MSC Xeno-Free Culture Medium (cat. no. Y50200, Cellartis; Takara Bio Europe $\mathrm{AB}$ ), EVs were prepared using an Exosome Extraction kit (cat no. E1310, Beijing Weihui Biotechnology Co., Ltd.) following the manufacturer's protocol. Cells and cell debris were removed by centrifugation at $2,000 \mathrm{x} \mathrm{g}$ at $4^{\circ} \mathrm{C}$ for $10 \mathrm{~min}$. Subsequently, the EVs were fixed in $2.5 \%$ glutaraldehyde for $2 \mathrm{~h}$ at room temperature. Following embedding in epoxy-based resins at room temperature for $3 \mathrm{~h}$, the samples were sectioned into 70-nm slices and stained with $3 \%$ uranium acetate and $3 \%$ lead citrate at room temperature for $5 \mathrm{~min}$. The slides were observed via transmission electron microscopy (JEM-1230; JEOL, Ltd.) at $80 \mathrm{KV}$. The particle sizes of exosomes were measured using a Laser Particle Size Analyzer (LA-960; HORIBA Scientific).

Preparation of NSCs. A total of 5 pregnant rats (age, 15 days; weight, $300 \mathrm{~g}$ ) were anesthetized with isoflurane (5\%) and then decapitated. The fetal rats were sterilized with $75 \%$ ethanol for $30 \mathrm{~min}$ at room temperature. The hippocampus tissue was separated and immersed in Hanks' Balanced Salt solution (Thermo Fisher Scientific, Inc.). After being fully shredded, the hippocampus tissue was digested with $0.125 \%$ trypsin for $10 \mathrm{~min}$ and the reaction was terminated using Dulbecco's Minimum Essential Medium (Gibco; Thermo Fisher Scientific, Inc.). The cells were collected and suspended in fresh medium containing 10\% FBS (Hyclone; Cytivia) and cultured in a $5 \% \mathrm{CO}_{2}$ incubator at $37^{\circ} \mathrm{C}$.

Immunohistochemistry. The collected cells were fixed in 4\% paraformaldehyde for $30 \mathrm{~min}$ at room temperature. Endogenous peroxidase activity was blocked with 3\% (v/v) $\mathrm{H}_{2} \mathrm{O}_{2}$ for $5 \mathrm{~min}$ at room temperature. The slides were blocked in $5 \%$ bovine serum albumin (Hyclone, Thermo Fisher Scientific, Inc.) at room temperature for $2 \mathrm{~h}$ and incubated with a monoclonal anti-nestin primary antibody (1:200; cat no. OM264981; Omnimabs) overnight at $4^{\circ} \mathrm{C}$, followed by incubation with a horseradish peroxidase (HRP)-labeled goat anti-rabbit IgG secondary antibody (1:10,000; cat. no. A16104SAMPLE; Thermo Fisher Scientific, Inc.) for $30 \mathrm{~min}$ at room temperature. Immunohistochemical staining was visualized using 3,3'-diaminobenzidine chromogen for $3 \mathrm{~min}$ at room temperature. Images were captured using a routine light microscope (magnification: 200x; BX51; Olympus Corporation) and at $\geq 5$ fields in each slide were selected at random.

Experimental groups. Following culture for $24 \mathrm{~h}$, the hypoxic injury model was established using $200 \mu \mathrm{M}$ cobalt chloride (purity: 97\%; Sigma-Aldrich; Merck KGaA), as previously described (21). The NSCs were divided into three groups: i) Normal NSCs group (Control); ii) NSCs hypoxic injury group (Model); and iii) NSCs hypoxic injury + MSCs-EVs group. Following treatment with cobalt chloride for $24 \mathrm{~h}$ at $37^{\circ} \mathrm{C}$, MSCs-EVs $(20 \mu \mathrm{g} / \mathrm{ml})$ were added to the cells $\left(3 \times 10^{5} / \mathrm{ml}\right)$ for a further $24 \mathrm{~h}$ at $37^{\circ} \mathrm{C}$. To evaluate the effect of miR-210-3p, an miR-210-3p inhibitor was designed and transfected into NSCs. The NSCs were divided into four groups: i) Control group; ii) hypoxic injury group (Model); iii) hypoxic injury + miR-210-3p NC group (miR-210-3p NC); and iv) hypoxic injury + miR-210-3p inhibitor group (miR-210-3p inhibitor).

Cell transfection. At $70 \%$ confluence, NSCs were transfected. The transfection solution consisted of $125 \mu \mathrm{lOpti}-\mathrm{MEM}$ (Gibco, Thermo Fisher Scientific, Inc.), $5 \mu 1$ Lipofectamine ${ }^{\circledR} 3000$ (Thermo Fisher Scientific, Inc.) and $12.5 \mu 1$ RNA inhibitor (RNA inhibitor powder dissolved in diethyl pyrocarbonate; $40 \mu \mathrm{g} / 125 \mu \mathrm{l})$. The transfection solution was added to the corresponding wells in the 6 -well plate for $4 \mathrm{~h}$ and the final concentration of miR-210-3p inhibitor was $1 \mu \mathrm{g} / \mathrm{ml}$. Subsequently, complete medium containing $20 \%$ FBS was added to the 6-well plate at $37^{\circ} \mathrm{C}$. Then, $48 \mathrm{~h}$ later at $37^{\circ} \mathrm{C}$, the transfections were verified by reverse transcription-quantitative PCR (RT-qPCR). miR-210-3p inhibitor was synthesized by Anhui General Bioengineering Co., Ltd. The sequences of miR-210-3p inhibitor and NC were UCAGCCGCUGUCACA CGCACAG (5'-3') and UCUACUCUUUCUAGGAGGUUG UGA (5'-3'), respectively.

TUNEL. NSC apoptosis was detected by performing a TUNEL assay (cat no. C1082; Beyotime Institute of Biotechnology) following the manufacturer's protocol. Briefly, terminal deoxynucleotidyl transferase $(2 \mu \mathrm{l})$ and fluorescent reagent (DAPI, $48 \mu \mathrm{l}$ ) were mixed and applied to the cells at $37^{\circ} \mathrm{C}$ for $60 \mathrm{~min}$. Following washing by $0.1 \mathrm{M}$ phosphate buffer saline, the slides were covered with anti-fading reagent and observed under a fluorescence microscope (magnification: 200x; BX51; Olympus Corporation) and $\geq 5$ fields in each slide were selected randomly.

Reverse transcription-quantitative PCR. Following treatment, total RNA was extracted from cells using an Ultrapure RNA extraction kit (CoWin Biosciences). The purity of RNA was assessed by measuring the optical density of each sample at a wavelength of $280 / 260 \mathrm{~nm}$ by a spectrophotometer (ShanghaiPuyuan Instrument Co., Ltd.). RNA $(1 \mu \mathrm{g})$ was reverse transcribed into cDNA using an Avian Myeloblastosis Virus Reverse-Transcriptase kit (cat. no. CW2141S; CoWin Biosciences). The qPCR reaction system included $7 \mu 1$ RNase-Free dH2O, $1 \mu \mathrm{l}$ cDNA/DNA, $2 \mu 1$ primers and $10 \mu 1$ 2XUltraSYBR Mixture (cat. no. 00081405; CoWin Biosciences). qPCR was performed using the following 


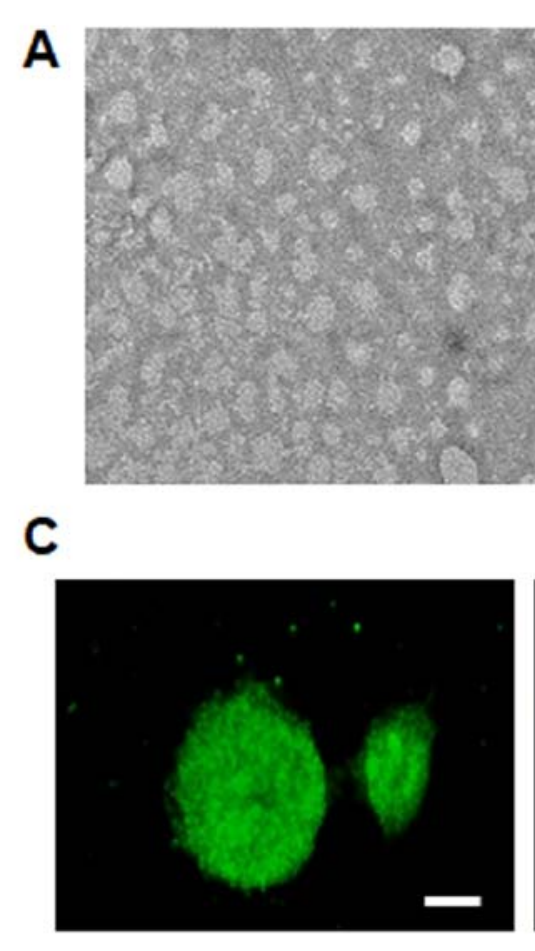

Nestin

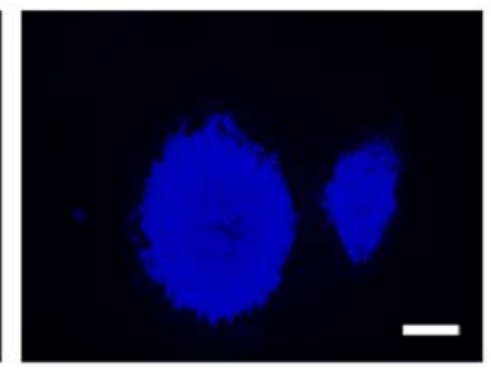

DAPI
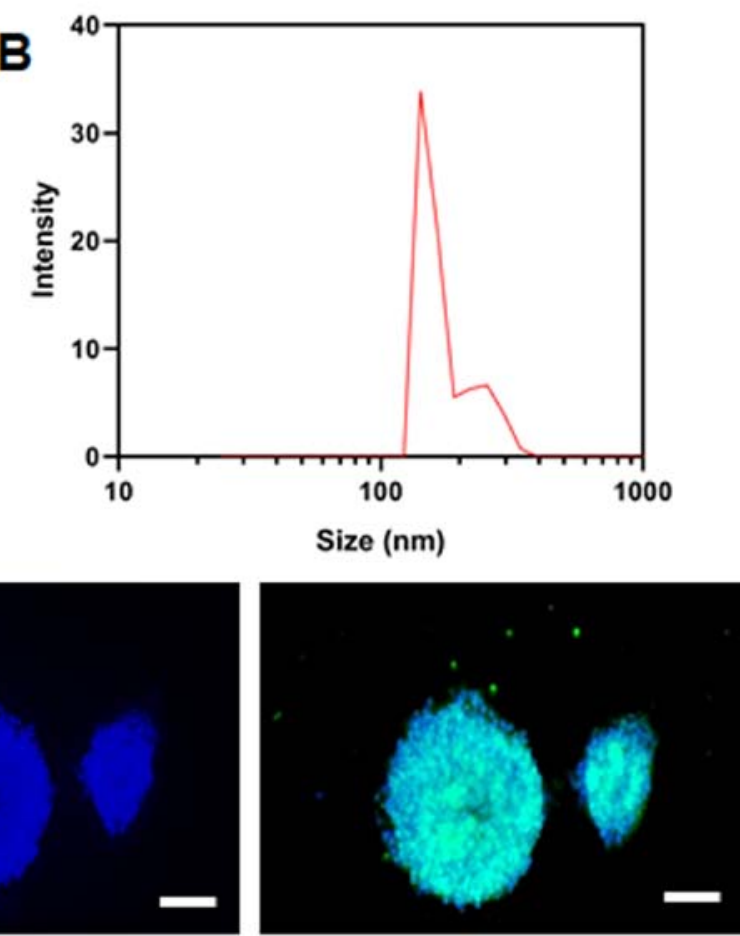

Merge

Figure 1. Identification of EVs and NSCs. (A) Morphology of MSC-EVs observed via transmission electron microscopy (scale bar, 200 nm). (B) Measurement of the particle size. (C) Identification of NSCs isolated from the hippocampus of embryonic Sprague-Dawley rats. Successful isolation of NSCs was indicated by $98 \%$ of the cells being nestin-positive (scale bar, $100 \mu \mathrm{m}$ ). EVs, extracellular vesicles; MSC, mesenchymal stem cell; NSCs, neural stem cells.

thermocycling conditions: Predenaturation for $10 \mathrm{~min}$; 40 cycles of denaturation at $95^{\circ} \mathrm{C}$ for $10 \mathrm{sec}$, annealing at $58^{\circ} \mathrm{C}$ for $30 \mathrm{sec}$ and extension at $72^{\circ} \mathrm{C}$ for $30 \mathrm{sec}$. The following primers were used for qPCR: U6 forward, 5'-GCTTCGGCA GCACATATACTAAAAT-3' and reverse, 5'-CGCTTCACG AATTTGCGTGTCAT-3'; and forward, rno-miR-210-3p, 5'-CTGTGCGTGTGACAGCGGCTGA-3'; reverse, 5'-GCA CTGCCTGCGGGACATACC-3'. The target gene expression was normalized to U6 using the $2^{-\Delta \Delta \mathrm{Cq}}$ method (23).

Western blotting. Following treatment, total protein was extractedfromcells using the TriplePrepkit(cat.no.28-9425-44; ReadyPrep; Cytiva). Total protein was quantified using a bicinchoninic acid protein assay kit (Beyotime Institute of Biotechnology). A total of $25 \mu \mathrm{g} /$ lane protein was separated via SDS-PAGE (10\% gel) and transferred onto nitrocellulose membranes, as previously described (24). The membranes were blocked in 5\% skimmed milk at room temperature for $2 \mathrm{~h}$ and incubated with the following primary antibodies overnight at $4^{\circ} \mathrm{C}$ : Rabbit polyclonal anti-Bcl-2 $19 \mathrm{kDa}$ interacting protein (BNIP3; 1:1,000; cat. no. ab109362; Abcam), mouse polyclonal anti-apoptosis-inducing factor (AIF; 1:1,000; cat. no. BF0591; Affinity Biosciences) and mouse monoclonal anti- $\beta$-actin (1:2,000; cat. no. TA890010; OriGene Technologies, Inc.). The membranes were washed three times with $1 \mathrm{X}$ phosphate-buffered saline with $0.05 \%$ Tween (PBST) and incubated with secondary antibodies (HRP-labeled goat anti-rabbit $\mathrm{IgG}$; cat. no. 65-6120; Thermo Fisher Scientific, Inc.; HRP-labeled goat anti-mouse IgG; cat. no. 31430; Thermo Fisher Scientific, Inc.) at $4^{\circ} \mathrm{C}$ for $2 \mathrm{~h}$. Protein bands were visualized using an electrochemiluminescence kit (Thermo Fisher Scientific, Inc.). Protein expression was quantified using the Quantity One software (v4.62; Bio-Rad Laboratories, Inc.) with $\beta$-actin as the loading control.

Statistical analysis. Data are presented as the mean \pm standard deviation with five experimental repeats in each group. One-way ANOVA followed by the Newman-Keuls post hoc test was used to analyze comparisons between multiple groups. Statistical analyses were performed using SPSS software (version 19.0; IBM Corp.). $\mathrm{P}<0.05$ was considered to indicate a statistically significant difference.

\section{Results}

Identification of EVs and hippocampal cells. EVs were imaged via transmission electron microscopy (Fig. 1A) and the results indicated that the particle size of EVs was $\sim 150-200 \mathrm{~nm}$ (Fig. 1B). The positive rate of nestin, a specific marker of NSCs, in the isolated cells was $>98 \%$ based on immunohistochemical staining (Fig. 1C), indicating the successful isolation of NSCs.

$M S C$-EVs reduce hypoxic injury-induced NSC apoptosis, and AIF and BNIP3 expression levels, but promote miR-210-3p expression. Compared with the control group, NSC apoptosis in the model group was significantly higher (Fig. 2). By 

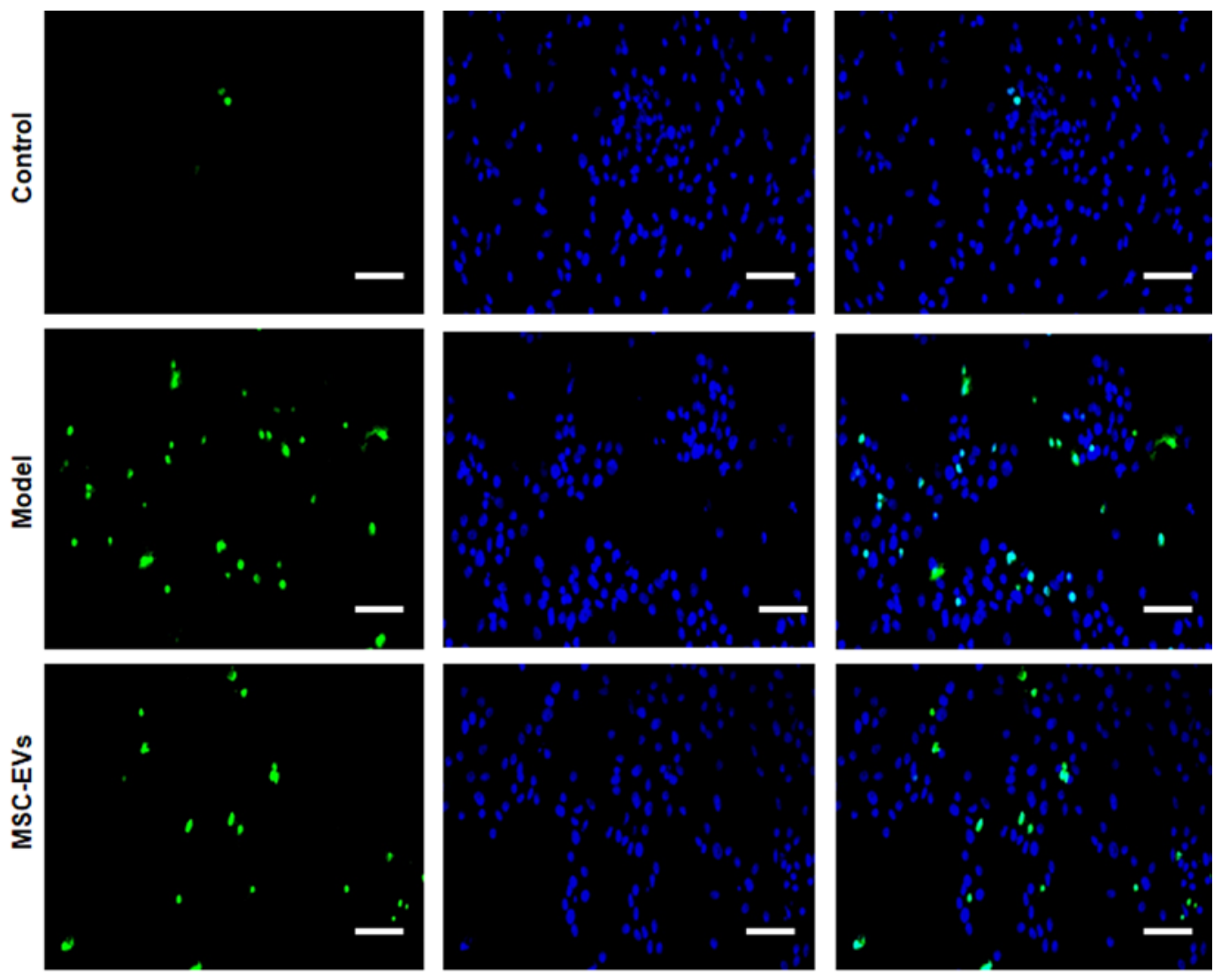

TUNEL

DAPI

Merge

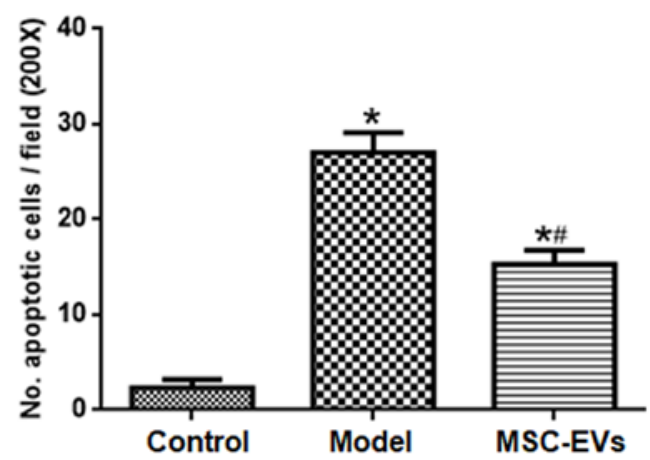

Figure 2. MSC-EVs reduce hypoxic injury-induced NSC apoptosis. NSC apoptosis was determined by performing a TUNEL assay (scale bar, $50 \mu \mathrm{m}$ ). ${ }^{*} \mathrm{P}<0.05$ vs. control; ${ }^{\#} \mathrm{P}<0.05$ vs. model ( $\mathrm{n}=5$ per group). MSC, mesenchymal stem cell; EVs, extracellular vesicles; NSC, neural stem cells.

contrast, NSC apoptosis was significantly reduced in the MSC-EVs group compared with the model group $(\mathrm{P}<0.05)$.

The expression levels of AIF and BNIP3 in the model group were significantly higher compared with the control group, whereas the expression levels of AIF and BNIP3 in the MSC-EVs group were significantly lower compared with the model group $(\mathrm{P}<0.05$; Fig. $3 \mathrm{~A})$.

Compared with the control group, miR-210-3p expression levels in the model group were significantly higher. In addition, treatment with MSC-EVs further increased miR-210-3p expression compared with the model group ( $\mathrm{P}<0.05$; Fig. $3 \mathrm{~B})$.

miR-210-3p inhibitor promotes hypoxic injury-induced NSC apoptosis, and AIF and BNIP3 expression levels. Compared with the control group, miR-210-3p expression was significantly reduced in miR-210-3p inhibitor-treated NSCs $(\mathrm{P}<0.05$;
Fig. 4A). Additionally, miR-210-3p inhibitor also reduced miR-210-3p expression levels compared with the model group $(\mathrm{P}<0.05$; Fig. 4B). In addition, compared with the model group, miR-210-3p inhibitor increased NSC apoptosis $(\mathrm{P}<0.05$; Fig. 5), and the expression levels of AIF and BNIP3 $(\mathrm{P}<0.05$; Fig. 6).

\section{Discussion}

The present study demonstrated that MSC-EVs reduced hypoxia injury-induced NSC apoptosis and promoted miR-210-3p expression. miR-210-3p inhibitor also promoted hypoxia injury-induced NSC apoptosis. Collectively, the results indicated that MSC-EVs possibly reduced apoptosis by modulating the miR-210-3p/AIF/BNIP3 signaling pathway. 
A

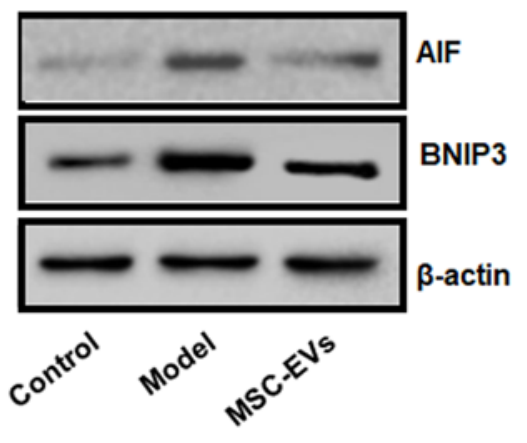

B

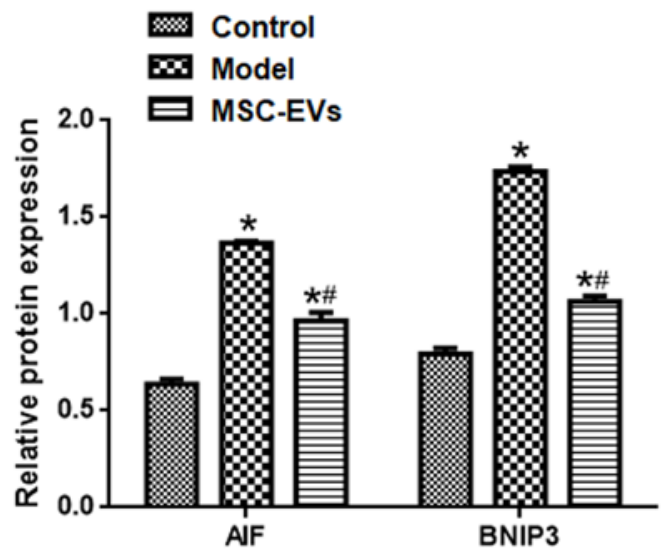

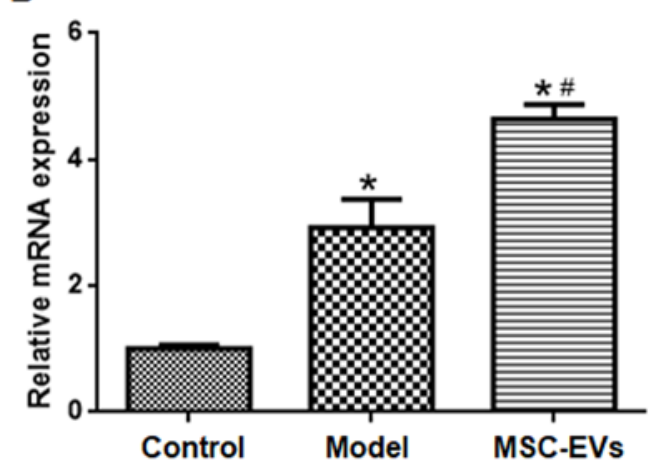

Figure 3. MSC-EVs reduce AIF and BNIP3 expression levels, but promote miR-210-3p expression levels in hypoxic injury-induced NSCs. (A) AIF, BNIP3 and (B) miR-210-3p expression levels. " $\mathrm{P}<0.05$ vs. control; " $\mathrm{P}<0.05$ vs. model ( $\mathrm{n}=5$ per group). MSC, mesenchymal stem cell; EVs, extracellular vesicles; AIF, apoptosis-inducing factor; BNIP3, Bcl-2 $19 \mathrm{kDa}$ interacting protein.
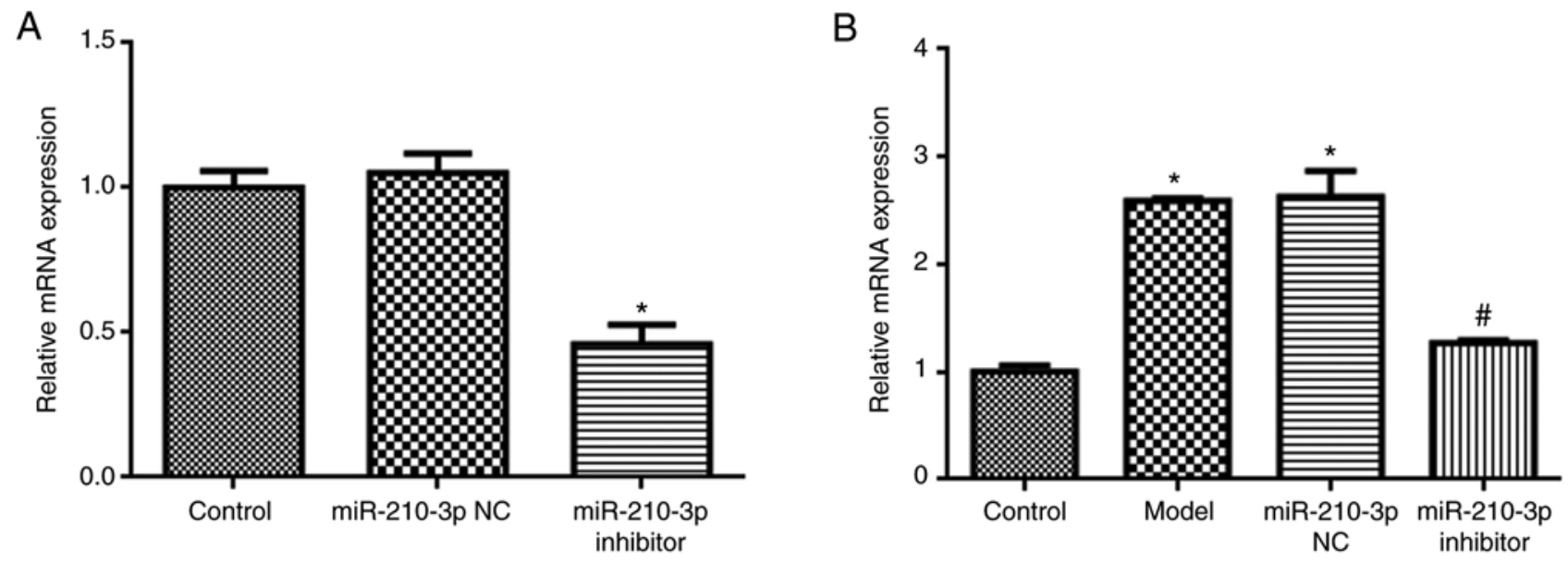

Figure 4. miR-210-3p inhibitor reduces miR-210-3p expression in NSCs. miR-210-3p expression in (A) normal and (B) hypoxic injured NSCs. "P<0.05 vs. control ; ${ }^{*} \mathrm{P}<0.05$ vs. miR-210-3p NC ( $\mathrm{n}=5$ per group). miR, microRNA; NSCs, neural stem cells; NC, negative control.

Cobalt chloride triggered NSC apoptosis and caused NSC hypoxic injury $(21,22)$. The signaling pathways involved in NSC apoptosis have been investigated; Mfat-1 transgene protects NSCs against cobalt chloride-mediated hypoxic injury by activating Nrf2/ARE signaling pathways (21). The present study demonstrated that MSC-EVs also protected NSCs against cobalt chloride-induced NSC apoptosis. MSCs are beneficial for a variety of diseases including stroke and infection diseases, primarily via self-differentiation and secretory vesicles (25), especially EVs $(26,27)$. EVs reach the injured area more easily than MSCs, as they can pass tissue barriers, such as the blood-spinal cord barrier, with ease (28). In addition, EVs are not susceptible to degradation in comparison with other biological products (29-31); therefore, EVs may serve as therapeutic agents for a range of diseases.

In the present study, EVs were extracted from MSCs, and the transmission electron microscopy and particle size analysis results indicated that the EVs were $>100 \mathrm{~nm}$ in size, which indicated the concentration and the purity of the extracted exosomes. NSCs were extracted from the hippocampus and 

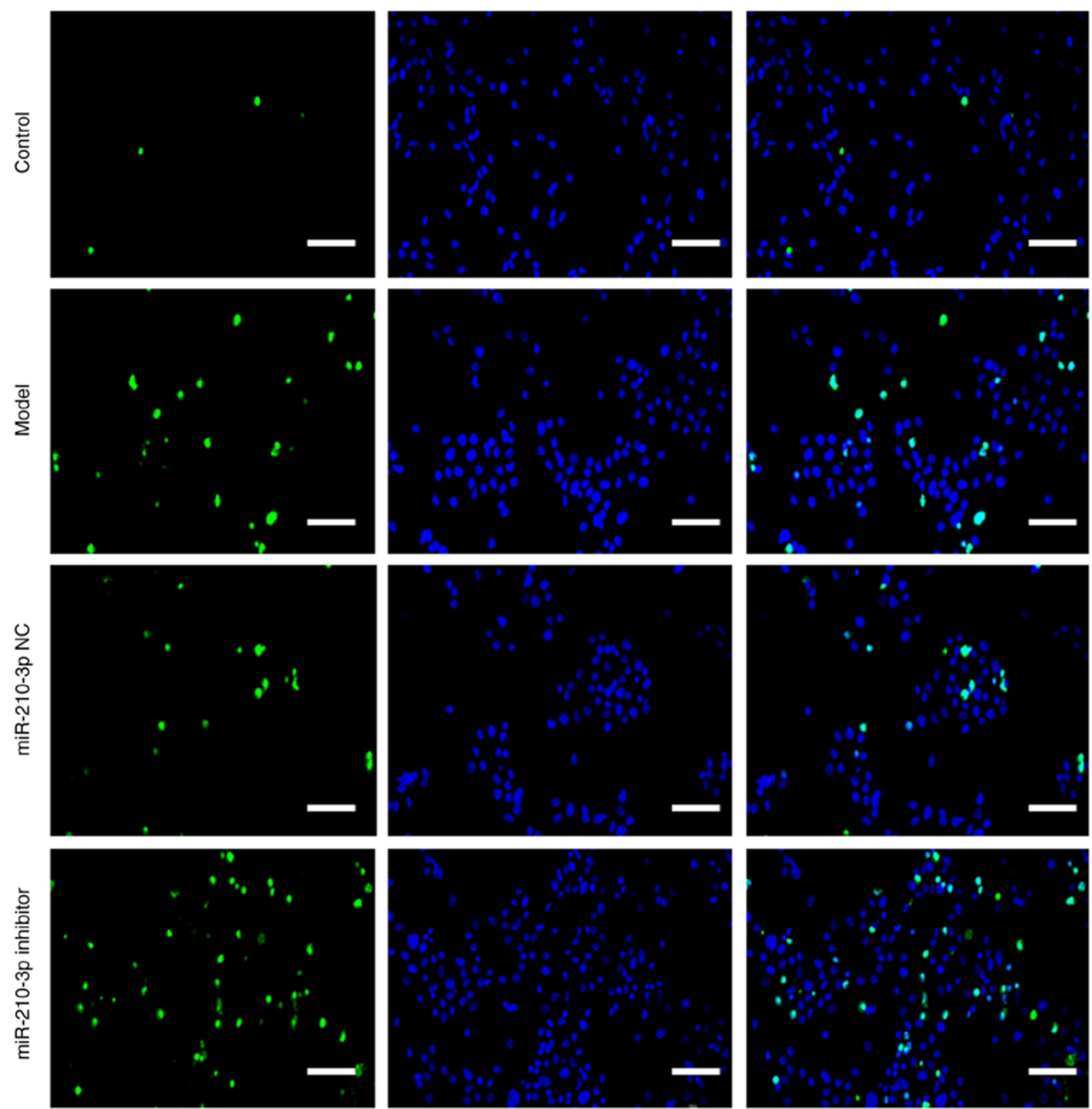

DAPI

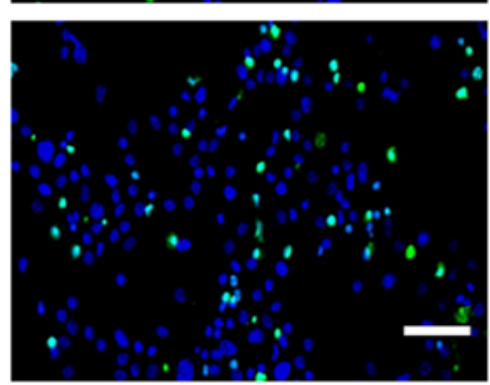

TUNEL

Merge

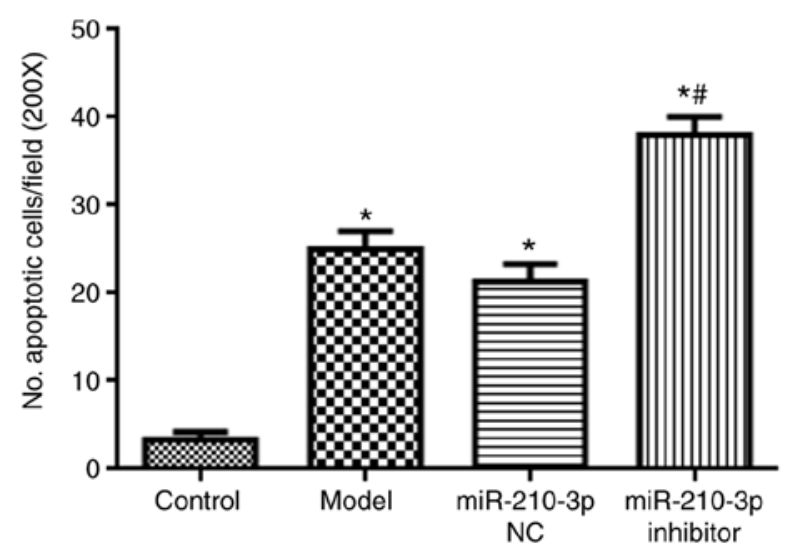

Figure 5. miR-210-3p inhibitor promotes hypoxia injury-induced NSC apoptosis. NSC apoptosis was determined by performing a TUNEL assay (scale bar, $50 \mu \mathrm{m})$. ${ }^{\mathrm{P}}<0.05$ vs. control; ${ }^{\#} \mathrm{P}<0.05$ vs. model ( $\mathrm{n}=5$ per group). miR, microRNA; NSCs, neural stem cells; NC, negative control.

cultured in vitro. The results suggested that the positive rate of nestin, a specific protein of neural stem cells, was $>98 \%$ in the extracted cells, which indicated that the isolation of NSCs from the hippocampus of SD rats was successful. A previous study indicated that exosome therapy can reduce the permeability of the blood-spinal cord barrier following spinal cord injury (32). The results of the present study also suggested that NSC apoptosis was increased in the hypoxic injury 

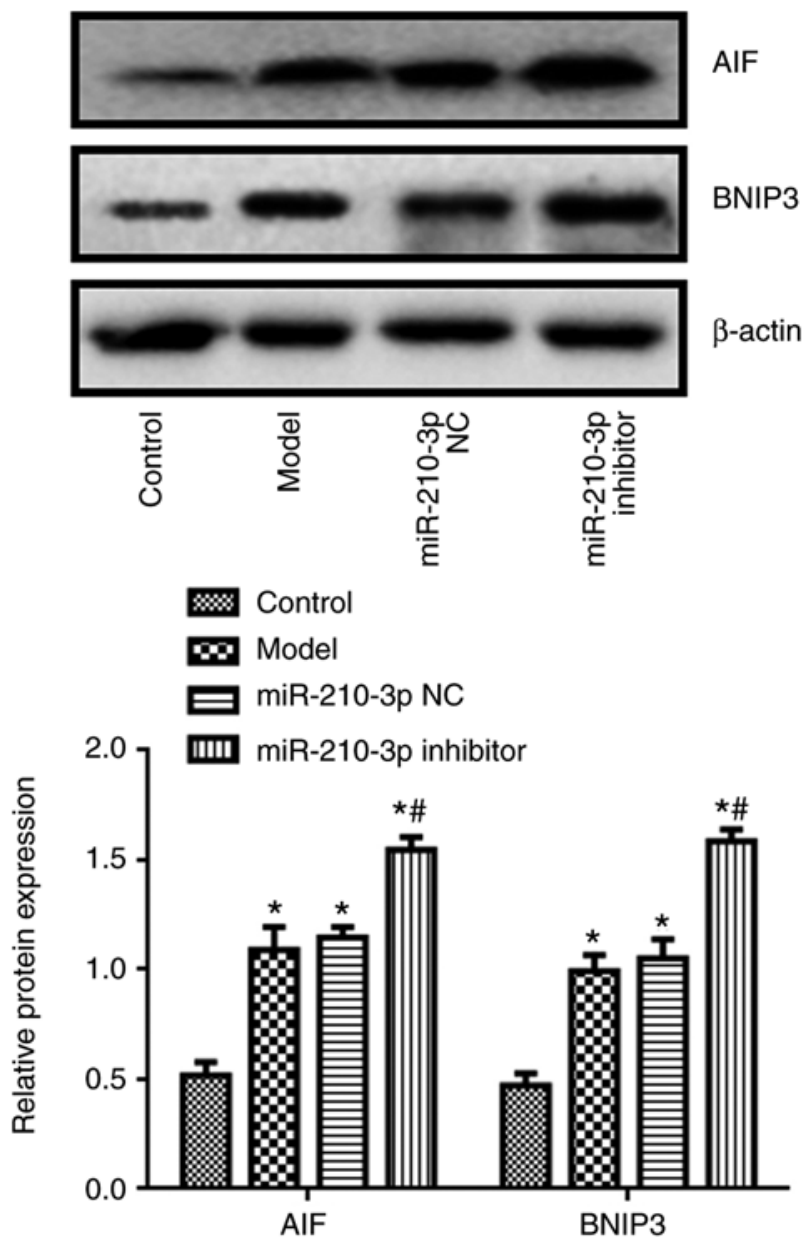

Figure 6. miR-210-3p inhibitor promotes hypoxia injury-induced AIF and BNIP3 expression in NSCs. miR-210-3p expression levels were determined by western blotting and quantified. ${ }^{*} \mathrm{P}<0.05$ vs. control; ${ }^{\#} \mathrm{P}<0.05$ vs. model ( $\mathrm{n}=5$ per group). miR, microRNA; AIF, apoptosis-inducing factor; BNIP3, Bcl-2 $19 \mathrm{kDa}$ interacting protein.

model group compared with the control group, but decreased following treatment with MSC-EVs.

The present study indicated that miR-210-3p expression was upregulated under hypoxic conditions compared with control conditions. miR-210-3p is an important post-transcriptional regulator that can inhibit MAX network transcriptional repressor expression, which is closely related to hypoxia (33). miR-210-3p can be activated by hypoxia inducible factor- $1 \alpha$ to promote cell survival (34). Reducing miR-210-3p expression antagonizes oxygen-glucose deprivation-induced injury (35). miR-210-3p is also an important regulator of cell apoptosis and proliferation (36). The present study revealed that MSC-EVs further promoted miR-210-3p expression in hypoxia injured NSCs and elevated miR-210-3p expression was beneficial for cell survival. miR-210-3p expression levels were decreased following treatment with miR-210-3p inhibitor in normal NSCs, as well as in hypoxia injured NSCs compared with control NSCs. Moreover, miR-210-3p inhibitor promoted hypoxic injury-induced NSC apoptosis.

The Bcl-2 family is involved in the process of cell injury (37). BNIP3 protein is the most sensitive apoptotic protein to hypoxia (38). BNIP3-induced cell death is characterized by increased plasma membrane permeability and mitochondrial damage in the early stages, but is not accompanied by caspase activation and cytochrome c release (39). AIF is normally located in the mitochondrial membrane gap, but during the stress reaction, AIF is released from mitochondria to cytoplasm and then transferred to nucleus (40). In the nucleus, AIF activates endogenous nucleic acid endonuclease (41). AIF-dependent cell death cannot be reduced by caspase inhibitors, suggesting that AIF does not depend on caspases (42). Under hypoxic conditions, the expression of miR-210-3p in NSCs is upregulated (41). miR-210-3p overexpression can promote NSC survival and proliferation and its mechanism may be related to BNIP3 (43). The present study revealed that the expression levels of BNIP3 and AIF in NSCs cells were decreased following treatment with MSC-EVs, but increased by miR-210-3p inhibitor compared with the model group. miR-210 can target hypoxia-inducible factor $1 \alpha$ to protect renal cells against hypoxia-induced apoptosis (34). miR-210-3p regulates non-small cell lung cancer cell proliferation and apoptosis by targeting paired amphipathic helix protein SIN3 transcription regulator family member A (44). miR-210-3p also targets repulsive guidance molecule A to enhance the angiogenic functions of endothelial progenitor cells under hypoxic conditions (45). However, whether miR-210-3p directly regulates BNIP3 and AIF is not completely understood.

The present study had a number of limitations. First, surface markers, such as cluster of differentiation (CD)9, CD81 and tumor susceptibility 101 were not detectable in the MSC-EVs. In the present study, the sizes of the prepared MSC-EVs were $>100 \mathrm{~nm}$, suggesting that these EVs are microvesicles (46), which typically contain CD40 as well as cholesterol, sphingomyelin, and ceramide. Therefore, these surface markers should be detected using sensitive assay methods in subsequent studies. Secondly, the present study suggested that MSC-EVs enhanced miR-210-3p expression, but whether MSC-EVs directly altered miR-210-3p expression was not investigated.

In summary, MSC-EVs prevented NSC hypoxic injury by promoting miR-210-3p expression and reducing apoptosis. Therefore, MSC-EVs may serve as an important treatment strategy for NSC hypoxic injury.

\section{Acknowledgements}

Not applicable.

\section{Funding}

The present study was supported by the Basic and Application Research in Yunnan Province [grant no. 2017FE468(-251)].

\section{Availability of data and materials}

The datasets used and/or analyzed during the current study are available from the corresponding author on reasonable request.

\section{Authors' contributions}

FL, JZ, RL, YD, LT and YX performed the experiments and analyzed the data. JZ and AC designed the study and wrote 
the manuscript. All authors read and approved the final manuscript.

\section{Ethics approval and consent to participate}

All experimental protocols were approved by the Ethics Committee of Kunming Medical University (approval no. KMMU2020180).

\section{Patient consent for publication}

Not applicable.

\section{Competing interests}

The authors declare that they have no competing interests.

\section{References}

1. Ma DK, Bonaguidi MA, Ming GL and Song H: Adult neural stem cells in the mammalian central nervous system. Cell Res 19: 672-682, 2009.

2. Vishwakarma SK, Bardia A, Tiwari SK, Paspala SA and Khan AA: Current concept in neural regeneration research: NSCs isolation, characterization and transplantation in various neurodegenerative diseases and stroke: A review. J Adv Res 5: 277-294, 2014.

3. Babenko VA, Silachev DN, Popkov VA, Zorova LD, Pevzner IB, Plotnikov EY, Sukhikh GT and Zorov DB: Mirol enhances mitochondria transfer from multipotent mesenchymal stem cells $(\mathrm{mmsc})$ to neural cells and improves the efficacy of cell recovery. Molecules 23: 687, 2018.

4. Zahr SK, Yang G, Kazan H, Borrett MJ, Yuzwa SA, Voronova A, Kaplan DR and Miller FD: A Translational repression complex in developing mammalian neural stem cells that regulates neuronal specification. Neuron 97: 520-537 e6, 2018

5. Xu X, Warrington AE, Bieber AJ and Rodriguez M: Enhancing CNS repair in neurological disease: Challenges arising from neurodegeneration and rewiring of the network. CNS Drugs 25 : 555-573, 2011.

6. Ullah I, Subbarao RB and Rho GJ: Human mesenchymal stem cells-current trends and future prospective. Biosci Rep 35: e00191, 2015.

7. Zhang X, Sai B, Wang F, Wang L, Wang Y, Zheng L, Li G, Tang J and Xiang J: Hypoxic BMSC-derived exosomal miRNAs promote metastasis of lung cancer cells via STAT3-induced EMT. Mol Cancer 18: 40, 2019.

8. Lee KW, Kim DH, Lee JH and Youn YN: The effect of pulsatile flow on bmsc-derived endothelial-like cells in a small-sized artificial vessel made by 3 -dimensional bioprinting. Stem Cells Int 2018: 7823830, 2018.

9. Chen L, Lu FB, Chen DZ, Wu JL, Hu ED, Xu LM, Zheng MH, Li H, Huang Y, Jin XY, et al: BMSCs-derived miR-223-containing exosomes contribute to liver protection in experimental autoimmune hepatitis. Mol Immunol 93: 38-46, 2018.

10. Wang Y, Zhao R, Liu D, Deng W, Xu G, Liu W, Rong J, Long X, $\mathrm{Ge} \mathrm{J}$ and Shi B: Exosomes derived from miR-214-enriched bone marrow-derived mesenchymal stem cells regulate oxidative damage in cardiac stem cells by targeting caMKII. Oxid Med Cell Longev 2018: 4971261, 2018.

11. Baglio SR, Rooijers K, Koppers-Lalic D, Verweij FJ, Pérez Lanzón M, Zini N, Naaijkens B, Perut F, Niessen HW, Baldini $N$ and Pegtel DM: Human bone marrow- and adipose-mesenchymal stem cells secrete exosomes enriched in distinctive miRNA and tRNA species. Stem Cell Res Ther 6: $127,2015$.

12. Su T, Xiao Y, Xiao Y, Guo Q, Li C, Huang Y, Deng Q, Wen J, Zhou $\mathrm{F}$ and Luo XH: Bone amesenchymal stem cells-derived exosomal MiR-29b-3p regulates Aging-associated insulin resistance. ACS Nano 13: 2450-2462, 2019.

13. Mead B and Tomarev S: Bone marrow-derived mesenchymal stem cells-derived exosomes promote survival of retinal ganglion cells through miRNA-Dependent mechanisms. Stem cells Transl Med 6: 1273-1285, 2017.
14. Santos JC, Lima NDS, Sarian LO, Matheu A, Ribeiro ML and Derchain SFM: Exosome-mediated breast cancer chemoresistance via miR-155 transfer. Sci Rep 8: 829, 2018.

15. Lin Y, Wu J, Gu W, Huang Y, Tong Z, Huang L and Tan J: Exosome-Liposome hybrid nanoparticles deliver CRISPR/Cas9 System in MSCs. Adv Sci (Weinh) 5: 1700611, 2018.

16. Michlewski G and Cáceres JF: Post-transcriptional control of miRNA biogenesis. RNA 25: 1-16, 2019.

17. Yang S, Mi X, Chen Y, Feng C, Hou Z, Hui R and Zhang W: MicroRNA-216a induces endothelial senescence and inflammation via Smad3/IkappaB $\alpha$ pathway. J Cell Mol Med 22: 2739-2749, 2018

18. Huang X, Le QT and Giaccia AJ: MiR-210-micromanager of the hypoxia pathway. Trends Mol Med 16: 230-237, 2010.

19. Ma X, Wang J, Li J, Ma C, Chen S, Lei W, Yang Y, Liu S, Bihl $\mathrm{J}$ and Chen $\mathrm{C}$ : Loading MiR-210 in endothelial progenitor cells derived exosomes boosts their beneficial effects on hypoxia/reoxygeneation-injured human endothelial cells via protecting mitochondrial function. Cell Physiol Biochem 46: 664-675, 2018.

20. Bavelloni A, Ramazzotti G, Poli A, Piazzi M, Focaccia E, Blalock W and Faenza I: MiRNA-210: A current overview. Anticancer Res 37: 6511-6521, 2017.

21. Yu J, Yang H, Fang B, Zhang Z, Wang Y and Dai Y: Mfat-1 transgene protects cultured adult neural stem cells against cobalt chloride-mediated hypoxic injury by activating Nrf2/ARE pathways. J Neurosci Res 96: 87-102, 2018.

22. Walls KC, Ghosh AP, Ballestas ME, Klocke BJ and Roth KA: Bcl-2/Adenovirus E1B 19-kd interacting protein 3 (BNIP3) regulates hypoxia-induced neural precursor cell death. J Neuropathol Exp Neurol 68: 1326-1338, 2009.

23. Livak KJ and Schmittgen TD: Analysis of relative gene expression data using real-time quantitative PCR and the 2(-Delta Delta C(T)) method. Methods 25: 402-408, 2001.

24. Song Z, Chen H, Xu W, Wu S and Zhu G: Basolateral amygdala calpain is required for extinction of contextual fear-memory. Neurobiol Learn Mem 155: 180-188, 2018.

25. Zuo R, Liu M, Wang Y, Li J, Wang W, Wu J, Sun C, Li B, Wang Z, Lan W, et al: BM-MSC-derived exosomes alleviate radiation-induced bone loss by restoring the function of recipient BM-MSCs and activating Wnt/ $\beta$-catenin signaling. Stem Cell Res Ther 10: 30, 2019.

26. Xu T, Xu M, Bai J, Lin J, Yu B, Liu Y, Guo X, Shen J, Sun H, Hao Y and Geng D: Tenocyte-derived exosomes induce the tenogenic differentiation of mesenchymal stem cells through TGF- $\beta$. Cytotechnology 71: 57-65, 2019.

27. Yu B, Shao H, Su C, Jiang Y, Xiteng Y, Bai L, Zhang Y, Li Q, Zhang X and Li X: Exosomes derived from MSCs ameliorate retinal laser injury partially by inhibition of MCP-1. Sci Rep 6: 34562, 2016.

28. Lu Y, Zhou Y, Zhang R, Wen L, Wu K, Li Y, Yao Y, Duan R and Jia Y: Bone mesenchymal stem cell-derived extracellular vesicles promote recovery following spinal cord injury via improvement of the integrity of the blood-spinal cord barrier. Front Neurosci 13: 209, 2019.

29. Gilligan KE and Dwyer RM: Engineering exosomes for cancer therapy. Int J Mol Sci 18: 1122, 2017.

30. Lou G, Song X, Yang F, Wu S, Wang J, Chen Z and Liu Y: Exosomes derived from miR-122-modified adipose tissue-derived MSCs increase chemosensitivity of hepatocellular carcinoma. J Hematol Oncol 8: 122, 2015.

31. Li X, Wang S, Zhu R, Li H, Han Q and Zhao RC: Lung tumor exosomes induce a Pro-inflammatory phenotype in mesenchymal stem cells via NFxB-TLR signaling pathway. J Hematol Oncol 9: 42, 2016.

32. Kong FL, Wang XP, Li YN and Wang HX: The role of exosomes derived from cerebrospinal fluid of spinal cord injury in neuron proliferation in vitro. Artif Cells Nanomed Biotechnol 46: 200-205, 2018.

33. Wang Z, Deng M, Liu Z and Wu S: Hypoxia-induced miR-210 promoter demethylation enhances proliferation, autophagy and angiogenesis of schwannoma cells. Oncol Rep 37: 3010-3018, 2017.

34. Liu LL, Li D, He YL, Zhou YZ, Gong SH, Wu LY, Zhao YQ, Huang X, Zhao T, Xu L, et al: miR-210 protects renal cell against hypoxia-induced apoptosis by targeting HIF-1 alpha. Mol Med 23: 258-271, 2017.

35. Costales MG, Haga CL, Velagapudi SP, Childs-Disney JL, Phinney DG and Disney MD: Small Molecule inhibition of microRNA-210 reprograms an oncogenic hypoxic circuit. J Am Chem Soc 139: 3446-3455, 2017 
36. Sun LL, Li WD, Lei FR and Li XQ: The regulatory role of microRNAs in angiogenesis-related diseases. J Cell Mol Med 22: 4568-4587, 2018

37. Tsujimoto Y: Role of Bcl-2 family proteins in apoptosis: Apoptosomes or mitochondria? Genes Cells 3: 697-707, 1998

38. Burton TR and Gibson SB: The role of Bcl-2 family member BNIP3 in cell death and disease: NIPping at the heels of cell death. Cell Death Differ 16: 515-523, 2009.

39. Jin Q, Li R, Hu N, Xin T, Zhu P, Hu S, Ma S, Zhu H, Ren J and Zhou H: DUSP1 alleviates cardiac ischemia/reperfusion injury by suppressing the Mff-required mitochondrial fission and Bnip3-related mitophagy via the JNK pathways. Redox Biol 14: 576-587, 2018

40. Bano D and Prehn JHM: Apoptosis-Inducing factor (AIF) in physiology and disease: The tale of a repented natural born killer. EBioMedicine 30: 29-37, 2018.

41. Liu J, Yuan C, Pu L and Wang J: Nutrient deprivation induces apoptosis of nucleus pulposus cells via activation of the BNIP3/AIF signalling pathway. Mol Med Rep 16: 7253-7260, 2017.

42. Benitez-Guzman A, Arriaga-Pizano L, Moran J and Gutierrez-Pabello JA: Endonuclease G takes part in AIF-mediated caspase-independent apoptosis in Mycobacterium bovis-infected bovine macrophages. Veterinary Res 49: 69, 2018.
43. Luan Y, Zhang X, Zhang Y and Dong Y: MicroRNA-210 protects PC-12 cells against hypoxia-induced injury by targeting BNIP3. Front Cell Neurosci 11: 285, 2017

44. Ren J, Li X, Dong H, Suo L, Zhang J, Zhang L and Zhang J: miR-210-3p regulates the proliferation and apoptosis of non-small cell lung cancer cells by targeting SIN3A. Exp Ther Med 18: 2565-2573, 2019.

45. Lu WJ, Liang HB, Li YF, Tu XQ, He JR, Ding KQ, Yang GY, Xin XY and Zeng LL: MicroRNA-210-3p targets RGMA to enhance the angiogenic functions of endothelial progenitor cells under hypoxic conditions. Front Cell Neurosci 13: 223, 2019.

46. Keshtkar S, Azarpira N and Ghahremani MH: Mesenchymal stem cell-derived extracellular vesicles: Novel frontiers in regenerative medicine. Stem Cell Res Ther 9: 63, 2018.

This work is licensed under a Creative Commons Attribution-NonCommercial-NoDerivatives 4.0 International (CC BY-NC-ND 4.0) License. 